\title{
Karyotyping human and mouse cells using probes from single-sorted chromosomes and open source software
}

Tamara A. Potapova1 , Jay R. Unruh¹, Andrew C. Box¹, William D. Bradford ${ }^{1}$, Christopher W. Seidel ${ }^{1}$, Brian D. Slaughter ${ }^{1}$, Shamilene Sivagnanam¹, Yuping Wu' $\mathrm{Wu}^{1}$, and Rong $\mathrm{Li}^{1,2, \dagger}$

${ }^{1}$ Stowers Institute for Medical Research, Kansas City, MO and ${ }^{2}$ Department of Molecular and Integrative Physiology, University of Kansas Medical Center, Kansas City, KS

${ }^{\dagger}$ Present address (R.L.): Department of Cell Biology, Johns Hopkins University School of Medicine, 855, N. Wolfe Street, Baltimore, MD 21205

BioTechniques 59:335-346 (November 2015) doi 10.2144/000114362

Keywords: karyotyping; spectral unmixing; chromosomes; aneuploidy; cytogenetics; Image J

Supplementary material for this article is available at www.BioTechniques.com/article/114362.

Multispectral karyotyping analyzes all chromosomes in a single cell by labeling them with chromosome-specific probes conjugated to unique combinations of fluorophores. Currently available multispectral karyotyping systems require the purchase of specialized equipment and reagents. However, conventional laser scanning confocal microscopes that are capable of separating multiple overlapping emission spectra through spectral imaging and linear unmixing can be utilized for classifying chromosomes painted with multicolor probes. Here, we generated multicolor chromosome paints from single-sorted human and mouse chromosomes and developed the Karyotype Identification via Spectral Separation (KISS) analysis package, a set of freely available open source ImageJ tools for spectral unmixing and karyotyping. Chromosome spreads painted with our multispectral probe sets can be imaged on widely available spectral laser scanning confocal microscopes and analyzed using our ImageJ tools. Together, our probes and software enable academic labs with access to a laser-scanning spectral microscope to perform multicolor karyotyping in a cost-effective manner.

Cytogenetics is an area of genomics focusing on chromosome number, structure, and composition, collectively referred to as a karyotype. Various karyotyping technologies can be used to detect structural chromosomal abnormalities, such as deletions, insertions, translocations, and numerical chromosomal aberrations (aneuploidy). Chromosomal abnormalities and aneuploidy have many pathological associations: Congenital somatic chromosome aneuploidy affects human development, causing embryonic lethality or developmental defects (1,2), and aneuploidy and other chromosomal aberrations constitute an established hallmark of cancer, with a majority of tumors displaying chromosomal gains, losses, and structural re-arrangements $(3,4)$. The karyotype defines the structural organization of the genome, and monitoring karyotypic changes provides important insights into gene expression and dynamics $(5,6)$. The development of karyotyping technology has enabled a greater understanding of chromosomal alterations in development and disease.

One important cytogenetics method is multicolor (multispectral or spectral) karyotyping. It is used in biomedical research and has clinical applications (7-9). Spectral karyotyping is instrumental in detecting whole chromosome gains and losses and can reveal structural aberrations of the chromosome, such as translocations, at the single-cell level. In this method, metaphase chromosomes are spread on a glass slide and hybridized to a cocktail of chromosome-specific fluorescent probes. Probes for DNA hybridization are typically generated by PCR amplification of individual chromosome pools separated by fluorescence activated sorting (FACS). These probes paint each chromosome with a unique combination of colors, making the chromosomes distinguishable from one another by microscopy imaging and analysis.

Discerning combinations of fluorochromes whose emission spectra can overlap is non-trivial and involves spectral imaging. Currently, two methods have been implemented to identify the spectral composition of chromosomes hybridized with multicolor probes. The first, multiplex fluorescence in situ hybridization (M-FISH), utilizes fluorochrome-specific optical filters

\section{METHOD SUMMARY}

Here we generated sets of chromosome paints from single-sorted human and mouse chromosomes for multispectral karyotyping that can be imaged on any spectral microscope. To complement this methodology, we also present a companion analysis package, Karyotype Identification via Spectral Separation (KISS), which is compatible with open source programs such as ImageJ or FIJI. 
$(10,11)$, while the second, spectral karyotyping (SKY), relies on interferometry (12). Image acquisition in $\mathrm{M}$-FISH involves imaging of each fluorescent color using specialized fluorochrome-specific narrow band-pass filters, and data are analyzed with M-FISH software that classifies each chromosome based on its fluorescent composition (10). The SKY interferometer method generates a fluorochrome-specific difference in optical path that provides spectral information about the object through a Fourier transform. Spectral emission data are analyzed using the software that classifies pixels in the multispectral image and performs karyotyping based on this classification (12).

Since both of these technologies were introduced in 1996, SKY has been widely used in research and medicine worldwide. However, SKY probes, along with the imaging and analysis platforms, are costly and are available commercially only from Applied Spectral Imaging. SKY probes for human and mouse are only available to SKY system owners. M-FISH modules, probes, and software are commercially available from the ZEISS Microscopy online store (www.micro-shop.zeiss.com/). Prohibitive costs of probes, equipment, and software often preclude academic researchers from using these technologies or stimulate outsourcing of karyotype analysis to service companies.

Over the past 20 years, multicolor fluorescence microscopes have been improved vastly. There has also been an increase in the variety of available fluorophores that can be utilized to label DNA. While the commercially available SKY and M-FISH systems utilize special filters and interferometer technology, modern advances in spectral imaging can make spectral karyotyping simpler, more efficient, and more accessible than ever before.

Here, we generated multicolor chromosome paint probes from singlesorted human and mouse chromosomes and employed spectral imaging and spectral unmixing to separate fluorescence emissions and identify chromosomes. We have also assembled a set of freely available, open source ImageJ plugins that perform chromosome segmentation, spectral unmixing, and karyotyping for human and mouse chromosomes (Figure 1A). Chromosome spreads painted with our multispectral probe sets can be imaged on various spectral laser scanning confocal

\section{A - Workflow}

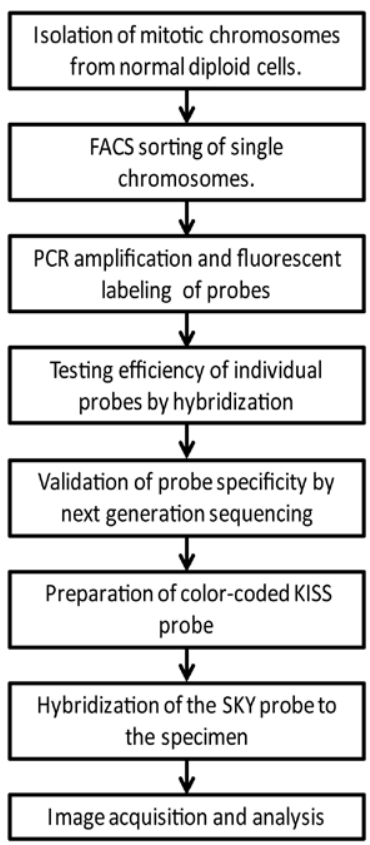

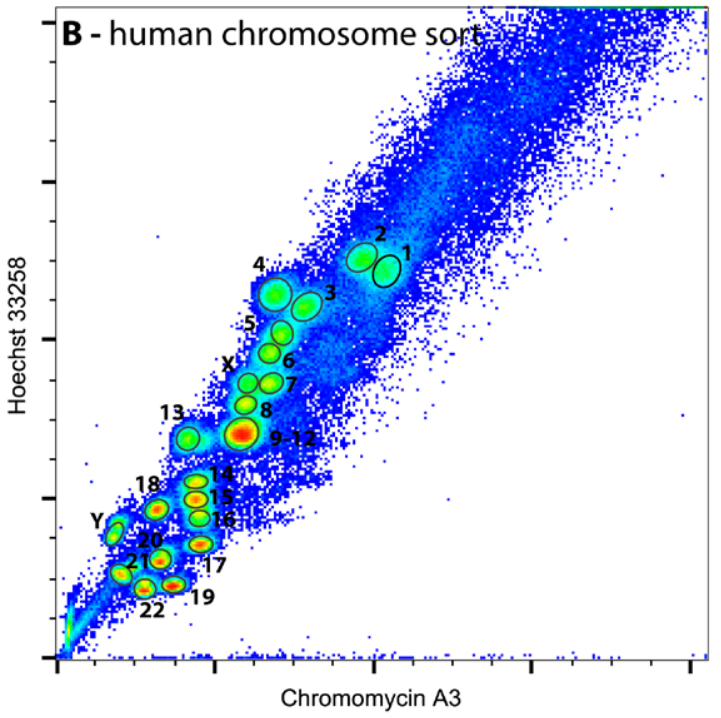

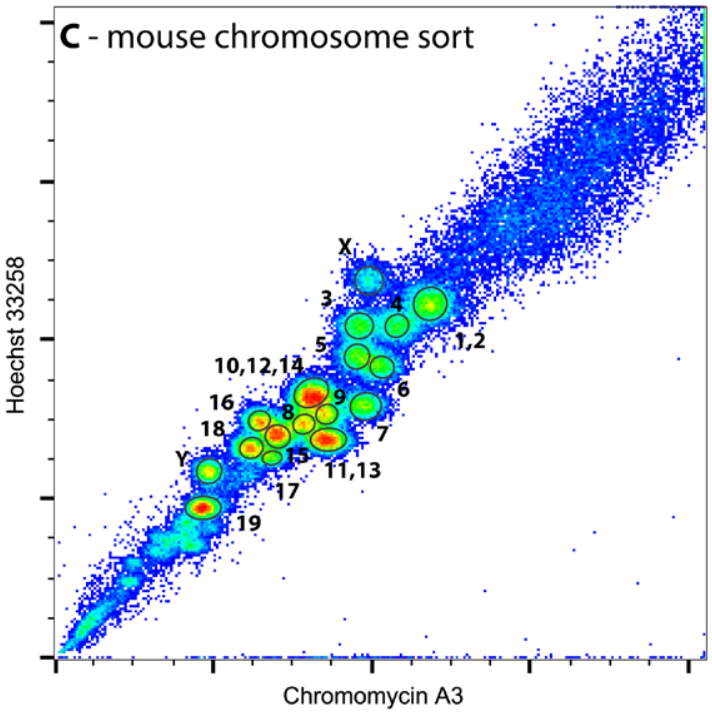

Figure 1. Classification and sorting of mitotic chromosomes from human and mouse cells by flow cytometry. (A) Diagram of workflow for multispectral probe generation and karyotyping. (B) Karyogram of chromosomes isolated from the human male Hs27 primary fibroblast cell line. (C) Karyogram of chromosomes isolated from the mouse feeder-independent E14Tg2a.4 embryonic stem cell line (male).

microscopes and then analyzed using ImageJ.

\section{Materials and methods}

single-chromosome sorting

To produce a cocktail of chromosomespecific, fluorescently labeled probes, we started by generating individual chromosome probes, which were subsequently combined into multicolor chromosome paints. Initially, individual probes were made from several hundred flow-sorted chromosomes as in References 13 and 14. However, these individual probes frequently cross-reacted with other chromosomes, indicating that the FACS-sorted populations were not suffi- ciently pure. To circumvent this problem, we utilized a single-chromosome sorting approach where only a single chromosome was sorted per tube for each PCR reaction. This approach precludes the possibility of contamination by other chromosomes and allows generation of highly specific probes (15). Human chromosomes used for sorting were isolated from the primary human fibroblast cell line Hs27 (Figure 1B), or the hTERTimmortalized diploid cell lines $\mathrm{CHON}-002$ (CRL-2847) and RPE1 (CRL-4000), both obtained from the American Type Culture Collection (ATCC) (Manassas, VA). Mouse chromosomes were isolated from a feederindependent embryonic stem cell line, E14 (Figure 1C), and from cultured splenocytes of B6 mice obtained from Stowers Institute 
A

\section{1 st Amp. 2nd Amp.}
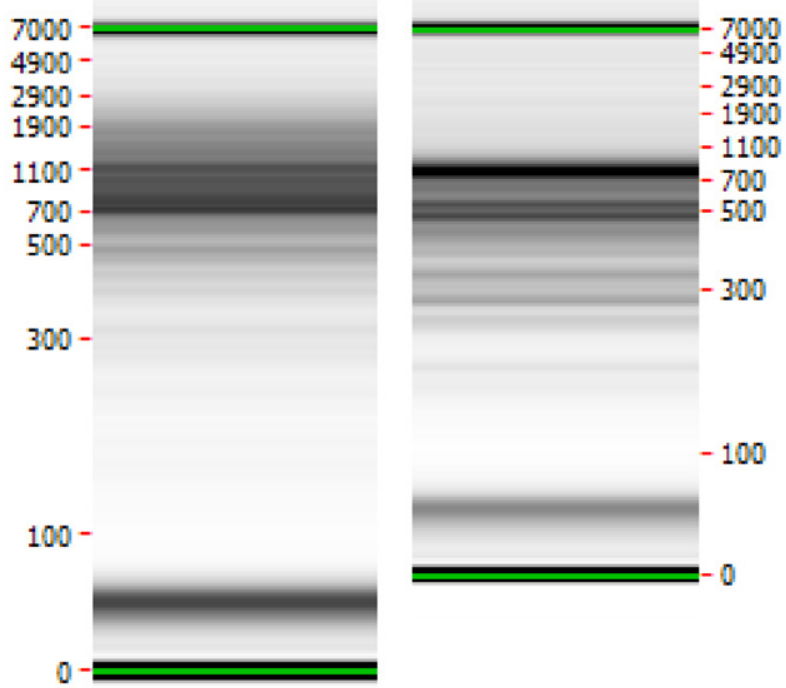

B

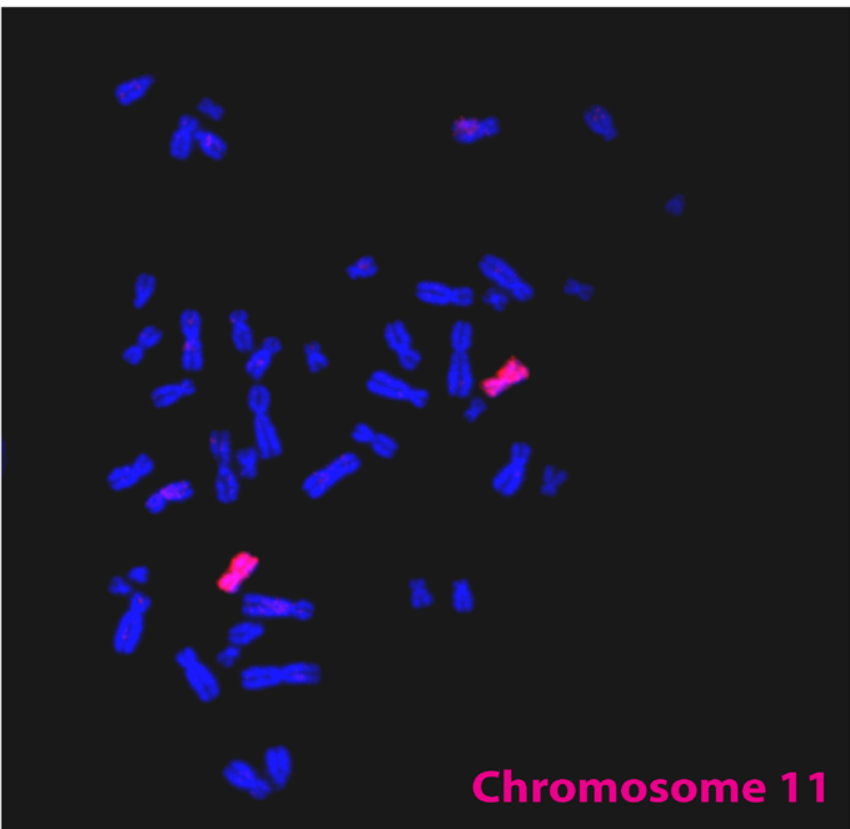

G

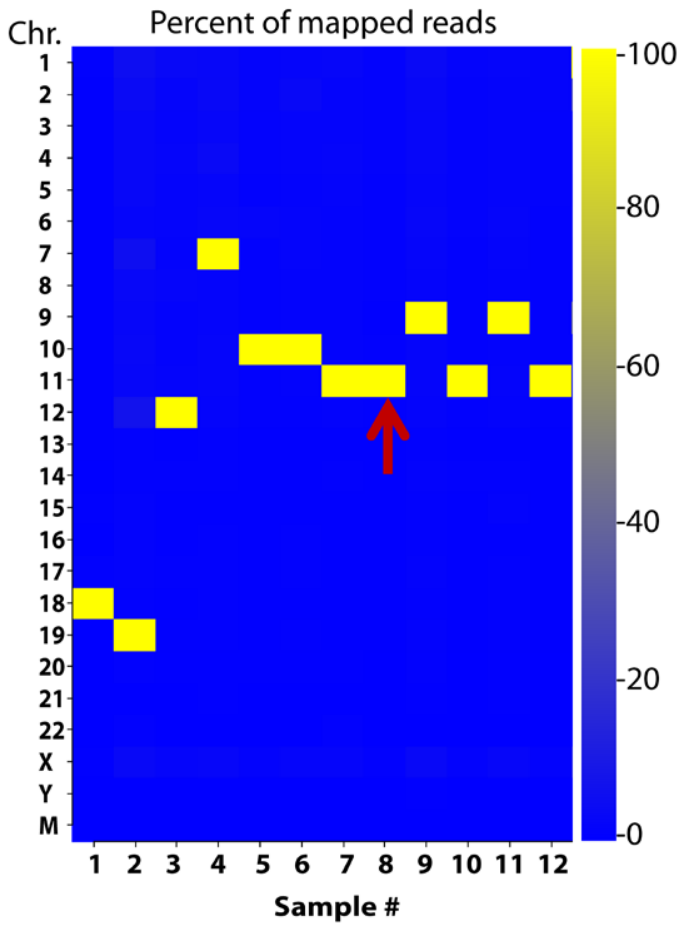

D

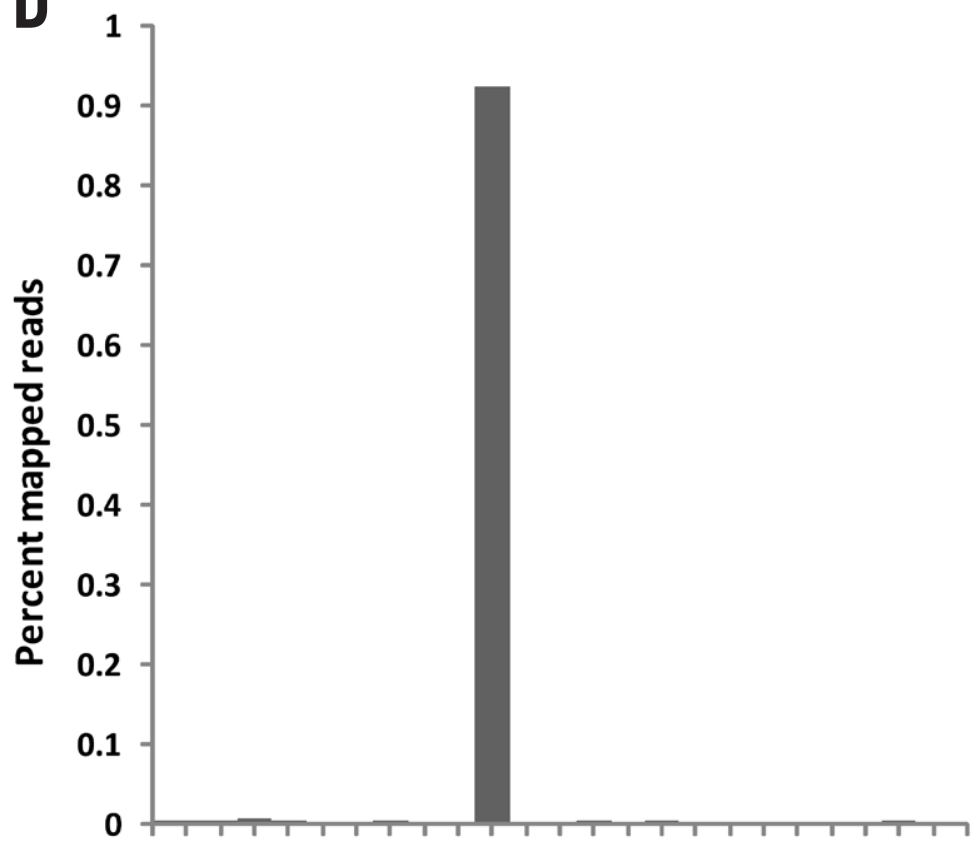

$12345678910111213141516171819202122 \times$ Y M

Human chromosome

Figure 2. Validation and reverse identification of single-sorted chromosomes by next-generation sequencing (NGS). (A) First and second degenerate oligonucleotide primed (DOP)-PCR amplifications of a human single-chromosome probe from the 9-12 chromosome cluster. Labchip electrophoresis showed good concentration of the PCR product in the 400-1000 bp range. (B) The probe in (A) was labeled by PCR with Atto-550 dUTP and hybridized to the mitotic chromosome spread from the human RPE1 cell line. The probe showed strong labeling of a pair of medium submetacentric chromosomes. (C) NGS of PCR probes from single-sorted chromosomes by short-read Illumina sequencing. The identities of single-sorted chromosome probes were determined by the chromosome with the highest fraction of mapped reads. The red arrow points to the probe shown in (A) and (B) (Sample \#8 in DNAseq), which was identified as chromosome 11. (D) Barplot representing fractions of mapped reads of individual Sample \#8, identified as chromosome 11.

animal facility (C57bl/6J mice originating from The Jackson Laboratory). Isolated chromosomes were stained with chromomycin A3 (cat.\# 80055-168; VWR, Radnor, PA) and Hoechst 33258 (cat.\# 14530; Sigma-Aldrich, St. Louis, MO) in polyamine- containing buffer. Hoechst 33258 has a propensity to bind AT bases and chromomycin A3 binds GC bases, which allowed resolution of double-stained chromosomes according to their base pair ratios and DNA content (Figure 1, B and C).
Amplification of single-sorted chromosome and validation by next-generation sequencing

Single-sorted chromosomes were amplified in two rounds of degenerate oligonucleotide primed (DOP) PCR (14) and labeled 
with dUTP conjugated to fluorochrome or biotin (Jena Bioscience GmbH, Jena, Germany). The first round of DOP-PCR amplified DNA from single-sorted chromosomes. First round amplifications were evaluated by electrophoresis. Successful first round amplifications that contained high concentrations (>30 $\mathrm{ng} / \mu \mathrm{l}$ ) of PCR product and increased proportions of high molecular weight products were amplified again to generate enough material for nextgeneration sequencing (NGS) reactions and fluorescent labeling. These probes were fluorescently labeled with dUTPs conjugated to Atto-550 (Jena Bioscience $\mathrm{GmbH}$ ). Fluorescently labeled PCR product (probe) was precipitated with ethanol and re-suspended in formamide hybridization solution. Each probe was tested individually by hybridization to human or mouse chromosome spreads from normal euploid cell lines. Probes that demonstrated strong and specific labeling were selected for validation by NGS. Sequence reads were mapped to the human genome using the short read alignment program bowtie (http://bowtie-bio.sourceforge.net) with parameters allowing up to 2 mismatches, and discarding reads that mapped to the genome more than once. The resulting BAM files were sorted, indexed, and summarized using samtools idxstats (www.htslib. org/) to attain mapped read counts per chromosome. The percentage of reads mapped to each human chromosome was calculated, plotted, and used to determine the identity of each probe (Figure 2). When all single-sorted chromosome probes were validated with NGS, multicolor labeling reactions were carried out to produce a cocktail of chromosome-specific, fluorescently labeled probes.

\section{Reverse identification of} clustered chromosomes

Not all human and mouse chromosomes could be resolved by FACS-sorting due to similarities in size and AT/GC content. For instance, human chromosomes 9, 10, 11, and 12 cluster as a single population (Figure 2A). Mouse karyotypes had three clusters that could not be resolved by FACS: 1 and 2; 10, 12, and 14; and 11 and 13 (Figure 1C). To generate chromosome-specific probes for these clusters, we used a reverse identification approach. First, chromosomes from these clusters were sorted into 96-well plates at one object per well and amplified by DOP-PCR (Figure 2A). Next, suitable probes were fluorescently labeled with dUTPs conjugated to Atto-550 dyes and tested by hybridization for quality labeling (Figure 2B). Probes that demonstrated the brightest labeling were selected for identification by next-generation DNA sequencing (DNAseq). For example, the probe shown in Figure 2, A and B was identified as chromosome 11 (Figure 2, C and D). After establishing the identity by DNAseq, probes were color-coded and used in multicolor chromosome paint.

\section{Dye selection and}

the chromosome code

Validated probes were labeled by PCR incorporation of dUTPs tagged with four spectrally distinct fluorescent dyes and biotin. Fluorescent dUTPs were conjugated with rhodamine green (A), and three Atto dyes (Jena Bioscience $\mathrm{GmbH}$ ): Atto532(B), Atto-550 (C), Atto-590 (D). Biotin was labeled with streptavidin conjugated to Cy5 (Jena Bioscience $\mathrm{GmbH}$ ). These fluorescent labels were selected based on their distinguishable emission spectra and the ability to be incorporated into PCR products by DNA polymerase. Each chromosome-specific painting probe was encoded by a unique combination of one to four dyes (Table 1).

Labeled probes were pooled and pre-annealed to mouse or human Cot-1 DNA containing sequences enriched for repetitive elements such as Alu repeats and centromeric DNA. Pre-annealing ensures that non-specific repetitive elements in the chromosome-painting probes are blocked from hybridizing to chromosome spreads. After the hybridization reaction, biotin was labeled with streptavidin conjugated to Cy5. DNA labeling with Hoechst 33342 was carried out as the last step before mounting the specimen. Our probe avoids digoxigenin antibody labeling step, which shortens the time needed for specimen preparation compared with the SKY method. Individual dyes for collecting spectra necessary for spectral unmixing were prepared in the same mounting medium as the specimen. A detailed protocol for labeling chromosome spreads is included in the Supplementary Material.

Spectral imaging and karyotype identification via spectral separation While both SKY and M-FISH technologies continue to use wide-field fluorescence microscopy, many types of microscopes capable of spectral imaging are available at present, including several confocal microscopes. In our study, laserscanning confocal microscopes LSM-710 and LSM-780 (Carl Zeiss Microimaging, Jena, Germany). Both microscopes were equipped with a QUASAR detection unit that can acquire with a single scan an entire range of emission wavelengths (in $10 \mathrm{~nm}$ increments) for subsequent spectral unmixing. For spectral imaging, 3 excitation laser lines were utilized: 488,561 , and $633 \mathrm{~nm}$. Images were collected with 3 different dichroics: the first passing $488 \mathrm{~nm}$ excitation, the second passing $488 \mathrm{~nm}$ and $561 \mathrm{~nm}$ excitation, and the third passing all 3 laser lines. In addition, a 405 nm laser was used to acquire a Hoechst 33342stained DNA image for segmentation, with emission collected at $\sim 450 \mathrm{~nm}$. All images were acquired with either a $40 \times$ or $63 \times$ Plan Apochromat objective (Carl Zeiss Microimaging). Pinhole settings were optimized for background reduction and signal-to-noise ratio.

Image processing and karyotyping were performed with a set of custom open source ImageJ ( $\mathrm{NIH}$, Bethesda, MD) plugins called Karyotype Identification via Spectral Separation (KISS), freely available at http:// research.stowers.org/imagejplugins/KISS_ analysis.html. Briefly, the plugins perform interactive background subtraction, spectral unmixing, interactive chromosome segmentation, and interactive karyotyping based on dye composition. Chromosome segmentation is performed using a semi-automated method based on the Hoechst image. First, the image is smoothed with a Gaussian blur with a 1 pixel standard deviation and then segmented with a manually chosen fractional threshold and object area limits to eliminate dirt and intact nuclei. Next, chromosomes too close to be separated by thresholding are manually separated. Finally overlapping chromosomes are separated into non-overlapping parts and then linked together for karyotyping.

Spectral unmixing was performed using the standard non-weighted linear least-squares method after subtraction of a manually selected background region. Spectral unmixing was performed on images excited with 488, 561, and 633 $\mathrm{nm}$ wavelengths, separately, with only the $500-x$ nm spectral region of the $488 \mathrm{~nm}$ image and the 560-x $\mathrm{nm}$ spectral region of the $633 \mathrm{~nm}$ image utilized for the unmixing. This avoids unnecessary interference from potential autofluorescent signal in those 
Table 1: Color code for multispectral chromosome probes for human and mouse.

\begin{tabular}{|c|c|c|c|c|c|c|}
\hline \multirow{2}{*}{$\begin{array}{c}\text { Human } \\
\text { Chromosome }\end{array}$} & \multicolumn{5}{|c|}{ Fluor. labeled dUTP } & \multirow{2}{*}{ LETTER CODE } \\
\hline & $\operatorname{RhG}(A)$ & A532(B) & $A 550(C)$ & A590(D) & Cy5(E) & \\
\hline Chr1 & & & & & & ABD \\
\hline Chr2 & & & & & & AE \\
\hline Chr3 & & & & & & ABE \\
\hline Chr4 & & & & & & CD \\
\hline Chr5 & & & & & & D \\
\hline Chr6 & & & & & & $A B C D$ \\
\hline Chr7 & & & & & & $A D$ \\
\hline Chr8 & & & & & & A \\
\hline Chr9 & & & & & & ABCE \\
\hline Chr10 & & & & & & ADE \\
\hline Chr11 & & & & & & B \\
\hline Chr12 & & & & & & BE \\
\hline Chr13 & & & & & & $A C D$ \\
\hline Chr14 & & & & & & BD \\
\hline Chr15 & & & & & & $\mathrm{DE}$ \\
\hline Chr16 & & & & & & $A B$ \\
\hline Chr17 & & & & & & ABDE \\
\hline Chr18 & & & & & & ACE \\
\hline Chr19 & & & & & & E \\
\hline Chr20 & & & & & & CE \\
\hline Chr21 & & & & & & BCD \\
\hline Chr22 & & & & & & C \\
\hline ChrX & & & & & & ACDE \\
\hline ChrY & & & & & & $A C$ \\
\hline Mouse & \multicolumn{5}{|c|}{ Fluor. labeled dUTP } & I FTTEP CODF \\
\hline Chromosome & $\operatorname{RhG}(A)$ & A532(B) & A550(C) & A590(D) & Cy5(E) & LEI IEK UUDL \\
\hline M Chr1 & & & & & & ABD \\
\hline M Chr2 & & & & & & $\mathrm{AE}$ \\
\hline M Chr3 & & & & & & ABE \\
\hline M Chr4 & & & & & & $C D$ \\
\hline M Chr5 & & & & & & D \\
\hline M Chr6 & & & & & & ABCD \\
\hline M Chr7 & & & & & & $A D$ \\
\hline M Chr8 & & & & & & DE \\
\hline M Chr9 & & & & & & B \\
\hline M Chr10 & & & & & & $\mathrm{BE}$ \\
\hline M Chr11 & & & & & & ACD \\
\hline M Chr12 & & & & & & BD \\
\hline M Chr13 & & & & & & BC \\
\hline M Chr14 & & & & & & $\mathrm{AB}$ \\
\hline M Chr15 & & & & & & A \\
\hline M Chr16 & & & & & & $\mathbf{E}$ \\
\hline M Chr17 & & & & & & $\mathrm{ABC}$ \\
\hline M Chr18 & & & & & & BCD \\
\hline M Chr19 & & & & & & C \\
\hline M ChrX & & & & & & ACE \\
\hline M ChrY & & & & & & AC \\
\hline \multicolumn{7}{|c|}{$\begin{array}{l}\text { Tables contain combinatorial color-code of five fluorescently labeled dUTPs used in human and mouse Karyo- } \\
\text { type Identification via Spectral Separation (KISS) probes. Five conjugates were used: rhodamine green, Atto- } \\
532 \text {, Atto-550, Atto-590, and biotin detected with streptavidin-Cy5. Colored boxes and the "Letter Code" } \\
\text { indicate which fluorescently labeled dUTPs comprise the probe for each chromosome. The KISS software } \\
\text { assigns chromosome identities by matching the individual contributions of the fluorophores with the code. }\end{array}$} \\
\hline
\end{tabular}

unneeded spectral regions. Note that the Atto-532 dye is excited at both 488 and 561 $\mathrm{nm}$. Similarly, the Atto-590 dye is excited at both $561 \mathrm{~nm}$ and $633 \mathrm{~nm}$. In both cases, the higher wavelength excitation result along with anti-stokes emission was used for further analysis due to lower autofluorescence contamination in these images.

Statistical analysis of the dye concentrations hybridized on each chromosome was assessed based on average unmixed intensities for the selected segmented chromosomes. Given the differential labeling, excitation, and detection efficiency of the dyes used, it was necessary to normalize the concentrations on an image-by-image basis. Given that at least four chromosomes were positive for each dye, the maximum and minimum dye concentrations were determined from the average of the highest and lowest four concentrations for each chromosome, respectively. In addition, we noticed that chromosomes were labeled with differential overall efficiency. Therefore all normalized fractional concentrations were divided by the strongest signal for that particular chromosome. The resulting fractional concentrations were compared with manually selected thresholds for each dye as well as the chromosome area to generate binary signal contributions for each chromosome. If these binary contributions are identical to the designed combinatorial code (Table 1), the chromosome is automatically assigned. Otherwise, chromosomes remain unassigned. The chromosomal code in the KISS software can be customized if probes are encoded with different combinations of colors than those shown in Table 1 (for more details see the software manual in the Supplementary Material).

False color images were also generated using the same normalization factors, but for each pixel individually. These pixel-bypixel concentrations were multiplied by the user-specified artificial dye colors for each dye channel to generate a composite image. Given the variegated nature of FISH probe labeling as well as the desire to avoid biased translocation assignment, we avoided automatic edge finding. Instead, we opted for an unbiased smoothing technique that performs Gaussian blurring only within the segmented objects. Alternatively, for noisy data and when translocations are not of interest, the user can average the concentration coloring uniformly over each entire chromosome. Colors can be toggled on and off individually for a more detailed analysis. 

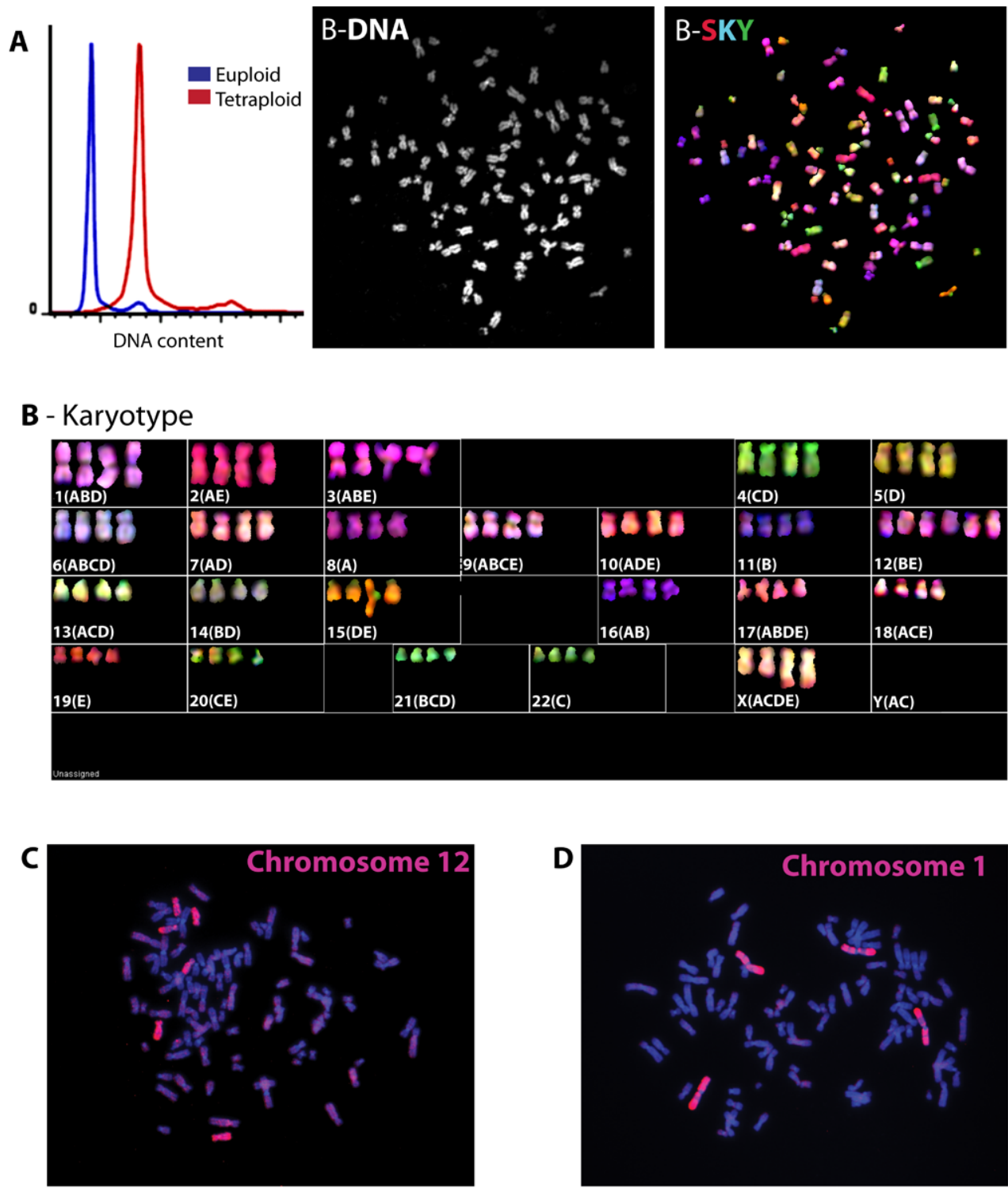

Figure 3. Karyotype of a tetraploid clonal derivative of the RPE1 cell line. (A) DNA content measured by flow cytometry in diploid (blue histogram) and tetraploid (red histogram) RPE1 cells. For this, asynchronously growing cells were collected by trypsinization, fixed in $20 \%$ ethanol, treated with RNase and stained with propidium iodide. The red histogram displays a shift toward a higher DNA content consistent with genome doubling. (B) Karyotype of a tetraploid derivative RPE1 cell line. Mitotic metaphase spreads were prepared from cells treated with colcemid for $8 \mathrm{~h}$ and processed as detailed in the Appendix A. Spectral karyotyping analysis showed tetraploidy, hexasomy of chromosome 12, and two aberrant $X$ chromosomes with an additional piece of chromosome 10 at the end of the q arm. This derivative $\mathrm{X}$ chromosome is present in the parental cell line as a single copy. (C) Confirmation of hexasomy 12 with singlechromosome-specific probe labeling validates the presence of six copies of chromosome 12. For the single-chromosome probe hybridization, metaphase spreads from tetraploid RPE1 cells were processed as for the spectral karyotyping and hybridized to the probe specific for chromosome 12. The probe was labeled with the Atto-550 dye. (D) Labeling of a tetraploid RPE1 chromosome spread with the probe specific for chromosome 1 confirms four copies of this chromosome. The probe was labeled with Atto-550 dye.
From this point onward, karyotyping proceeds manually based on false coloring, shape, and the normalized fractional contribution of each dye. To aid this process, the program can list possible matches in order of likelihood based on least-squares comparisons to the specified letter code. Finally, karyotype analyses can be saved to a ".kiss" file for further analysis or presentation. Alternatively, all images can be kept as standard ImageJ color images and further processed and saved for presentation or publication.

A detailed manual is provided in the Supplementary Material. In addition, the manual as well as sample human and mouse data are also available at http:// research.stowers.org/imagejplugins/ KISS_analysis.html.

\section{Results and discussion}

Using our spectral karyotyping system, we examined karyotypes from tetraploid clonal derivatives of the human RPE1 cell line (Figure 3). Tetraploidization, or genome doubling, is recognized as a prominent event of tumorigenesis in mammalian cells, because it could present an evolutionary step that allows tumors to become more genetically diverse and aggressive (16-21). RPE1 is a cell line derived from a normal human retinal pigmented epithelium cell culture immortalized with stable expression of human telomerase (22). This is a cell line of female origin containing 46 chromosomes, with one normal X-chromosome and one derivative $\mathrm{X}$-chromosome possessing an adjoined piece of chromosome 10 at the end of the q-arm. The tetraploid RPE1 clone shown in Figure 3 was derived from cultured cells transiently treated with an inhibitor of Aurora B kinase, followed by single-cell sub-cloning. Ploidy estimation by FACS analysis showed doubling of the DNA content (Figure 3A). However, counting chromosomes yielded the modal number of 94, indicating the presence of 2 additional chromosomes above the expected tetraploidy. Spectral unmixing and karyotyping showed six copies of chromosome 12, while the remaining somatic chromosomes displayed four copies. Our analysis also confirmed the derivative $X$ chromosome: two copies of $X$ showed an extra piece of chromosome 10 at the end of the q-arm, and the other two were normal $X$ chromosomes (Figure 3B). Hexasomy of chromosome 12 was confirmed with a single probe specific for human chromosome 12 


\section{A - DNA SEQ}
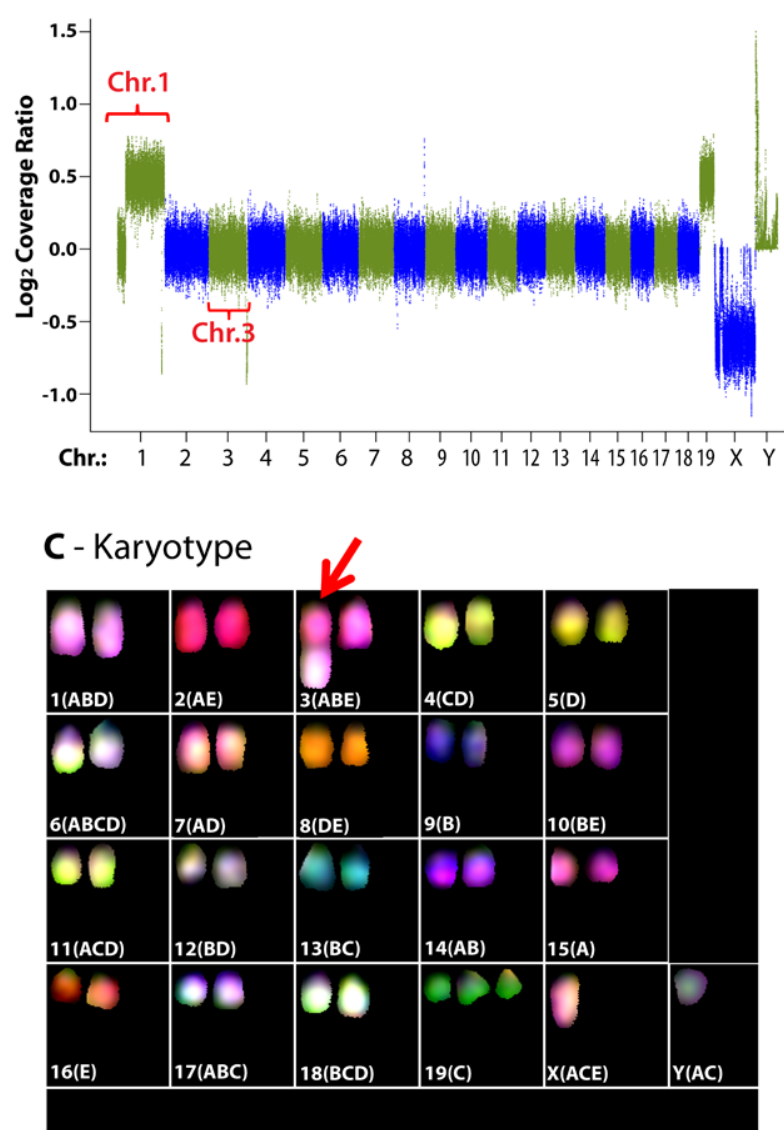
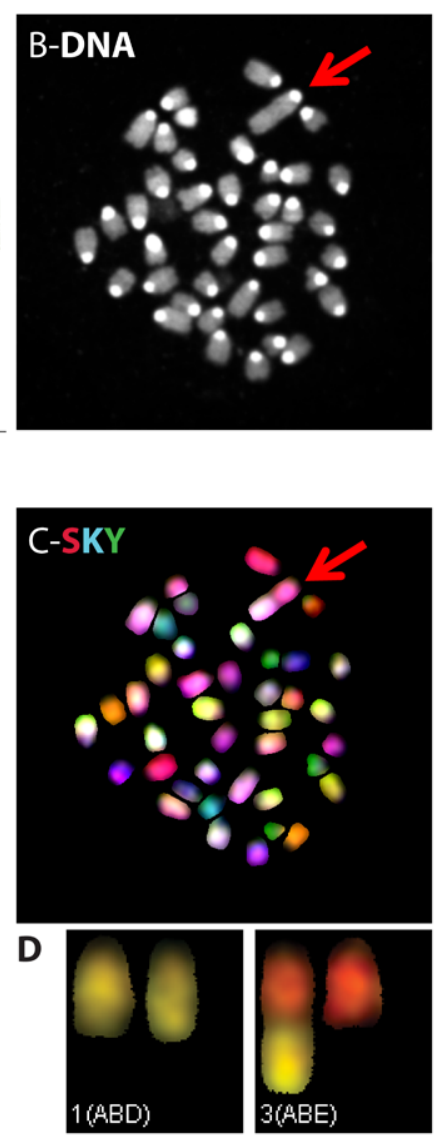

Figure 4. Karyotype of a mouse aneuploid clonal NSC line derived from primary neuronal stem cells. (A) Nextgeneration DNA sequencing (DNAseq) analysis of chromosome copy number. For this analysis, the number was compared with the euploid clone. The chromosome copy number was estimated from the fold-change (FC) ratio of normalized coverage of the aneuploid clone over the parental euploid clone. Reads from two chromosomes-chromosome 1 and chromosome 19-showed increased fold-change relative to the euploid NSC clone. A small region of chromosome 1 next to the centromere did not show an increased copy number, indicating a minute deletion. (B) Metaphase chromosome spread showing a chromosome with abnormally long arms (red arrow). (C) Karyotype of the aneuploid NSC cell showing chromosome 3 fused with extra chromosome 1 and trisomy 19. Mouse chromosome spreads were prepared from cells treated with colcemid for $4 \mathrm{~h}$ and processed as described for human samples in Figure 3B, except that the denaturation time was $1 \mathrm{~min}, 15 \mathrm{~s}$. A detailed description of methods is provided in the Supplementary Material. The extra-long chromosome highlighted in (B) is shown to be formed by a translocation of chromosome 1 on the $\mathrm{Q}$-arm of chromosome 3. In addition, there are three copies of chromosome 19. (D) An enlarged view of chromosomes 1 and 3 with channels $A, B$ and $\mathrm{C}$ toggled off highlights the chromosome 1 to chromosome 3 translocation. that was generated by single-chromosome sort and validated by NGS (Figure 3C). Hybridization of a probe specific for human chromosome 1 showed four copies of this chromosome, as expected (Figure 3D). This example demonstrates our KISS method is suitable for karyotyping human cells having abnormally high ploidy and chromosome numbers.

Next we determined the karyotypes of mouse aneuploid neuronal stem cell (NSC) lines. The presence of spontaneous aneuploidy in neuronal cells has been welldocumented (23-27), yet the functional significance of this phenomenon remains elusive. Here, aneuploid NSC clones were derived from primary NSCs isolated from embryonic mouse brain and propagated to form neurospheres. Neurospheres were then dissociated for ex vivo expansion to form single-cell clones. The ploidy of these clonal NSCs was first examined using mitotic chromosome counting, which showed aneuploidy in a fraction of the single-cell clones. The aneuploid NSC clone shown in Figure 4 has a chromosome number of 41 , as opposed to the normal chromosome number of 40 in a diploid mouse cell.
However, chromosome copy number analysis conducted by NGS indicated two extra chromosomes: chromosome 1 and chromosome 19 (Figure 4A). Moreover, according to the DNA sequencing data, a small region of chromosome 1 adjacent to the centromere did not show increased copy number, indicating a small deletion on one of the chromosomes (Figure 4A).

Examining the chromosome morphology in metaphase spreads identified one chromosome with aberrantly long arms (Figure 4B, arrow). Spectral karyotyping revealed this extra-long chromosome was actually a fusion between chromosome 3 and an additional chromosome 1 joined to the Q-arm of chromosome 3 (Figure 4, C and D). Our karyotyping also revealed an extra chromosome 19 in this aneuploid clone (Figure 4C). Therefore, even though this NSC clone contains 41 chromosomes, the actual chromosome copy number is increased for 2 chromosomes, chromosome 1 and chromosome 19, confirming the NGS findings. Consistent with the NGS data, our KISS method revealed that the extra chromosome 1 added onto chromosome 3 lost a centromere and a small range of the adjacent DNA sequence. Finally, chromosome 3 also showed a small subtelomeric deletion in one copy, indicating a breakpoint fusion event between a subtelomeric region of chromosome 3 and a nearcentromeric region of chromosome 1.

To test the utility of our karyotyping methodology for examining cancer karyotypes, we analyzed two cancer-derived cell lines: LoVo colon adenocarcinoma and $\mathrm{NCl}-\mathrm{H} 209$ small cell lung carcinoma. Instead of performing NGS, the karyotype of this cell line was determined by the DNA copy number information available through the Cancer Cell Line Encyclopedia (CCLE) at the Broad Institute (www.broadinstitute. org/ccle) (28). CCLE contains genetic information from a large panel of human cancer cell lines, including DNA copy number obtained by SNP array. CCLE data indicated increased copies of chromosomes 5, 7, 12, and 15 in the LoVo cell line (Supplementary Figure S1A), the same copy number result obtained by KISS analysis (Supplementary Figure S1B). The latter also showed two structural aberrations not discernable by the SNP array: a translocation of a piece of chromosome 2 onto chromosome 12 and 
a large metacentric isochromosome formed by fusion of two copies of chromosome 15 at the centromeric region.

The karyotype of the $\mathrm{NCl}-\mathrm{H} 209$ cell line (Supplementary Figure S2) was more complex, containing multiple re-arranged chromosomes and exhibiting cell-to-cell variation, indicating genomic instability. The CCLE DNA copy number analysis indicated gains and losses of multiple chromosomal segments (Supplementary Figure S2A). Karyotyping of this cell line using our KISS method revealed multiple structural re-arrangements. Two representative karyotypes are shown in Supplementary Figure S2B. Gains of large fragments of chromosomes 6, 7, and 8 corresponded with extra segments observed by the spectral karyotyping. Partial losses of chromosomes 1, 3, 4,5 , and 10 may be explained by structural rearrangements of these chromosomes. Karyotypes of both chromosomal spreads are detailed in the figure legend.

Multicolor karyotyping remains an important cytogenetic tool for discerning chromosome copy number variations and identifying segmental rearrangements of chromosomes on a single-cell basis. However, these methods are inadequate for identifying small structural genomic changes and can only be applied to actively dividing cells. Other, more recent array-based and sequencing-based molecular cytogenetics methods have improved detection of small numerical or structural chromosomal aberrations (29) and do not require mitotic cells, but these have their own limitations (30). Array-based Comparative Genomic Hybridization ( $\mathrm{CGH})$, has been widely used to detect whole chromosome or segmental aneuploidy. CGH arrays consist of immobilized nucleic acid sequences labeled with one fluorophore that are hybridized to complementary target sequences labeled with another fluorophore. Fluorescence ratios provide a representation of the relative DNA copy number. This method is sensitive, specific, and does not require mitotic cells. However, unlike multicolor karyotyping, it cannot detect genetic re-arrangements and does not provide single-cell resolution of the karyotype (31), which becomes an issue when the population is karyotypically heterogeneous.

Since the development of NGS, many karyotypic features can be determined by deep sequencing of the entire genome of the sample. This technology allows simulta- neous sequencing of the entire pool of DNA by producing sequence reads, which are then mapped to the reference genome (32). At sufficient genome coverage by sequence reads, NGS can detect even small genomic amplifications or deletions. The paired-end read mapping NGS technique also allows for detecting chromosomal rearrangements (31). Recently, it became possible to obtain genome-wide copy number information by NGS from single cells $(33,34)$. However, this approach cannot detect certain genome rearrangements, such as balanced translocations. At this time, NGS technology, especially on the singlecell level, is still demanding in terms of resources, equipment, and computational power.

Our results demonstrate the benefit of combining karyotyping and sequencing data to improve karyotype accuracy. Karyotyping by chromosome painting remains an indispensable cytogenetics tool, especially for single-cell analyses. However, the SKY method is costly, requiring the purchase of proprietary equipment and software in addition to painting probes. Our newly developed human and mouse probes are amenable to imaging on spectral micro-

\section{Virotag̊:}

\section{Direct Antibody-Based Virus Quantification in Minutes!}

The ViroTag ${ }^{\circledR}$ detection system provides mission-critical data in real-time.

\section{Eliminates the need for time-consuming plaque titer, qPCR and other methods when combined with the ViroCyt ${ }^{\circledR}$ Virus Counter $^{\circledR} 3100$.}

Immediate tracking of virus titer in your bioreactors to:

\section{- Optimize growth conditions}

- Maximize yields

- Identify problems sooner

Curious? Contact us at info@virocyt.com or visit www.virocyt.com to learn more!

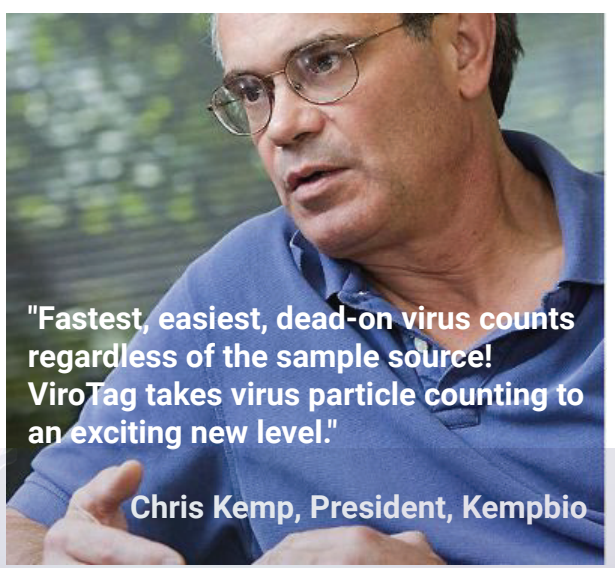


scopes, and the accompanying ImageJbased analysis tool offers robustness and flexibility in image acquisition and analysis. Together, these tools should allow academic labs, and potentially fertility and cancer clinics, to perform multicolor karyotyping in a more cost-effective manner.

\section{Author contributions}

T.A.P. designed the study and acquired, analyzed and interpreted data. J.R.U. conceived and designed the multispectral chromosome imaging and analysis and karyotyping software. A.C.B.implemented and fine-tuned the single chromosome isolation and sorting. W.D.B. implemented and fine-tuned the degenerate PCR. Christopher W. Seidel performed the next generation DNA sequencing and analysis. B.D.S. performed the multispectral image acquisition and analysis, and designed the chromosome color code. S.S. perfomred data analysis. Y.W. isolated mouse neuronal stem cells and generated aneuploid cell clones. R.Li. conceived the study and provided and intellectual contribution at all steps. All authors contributed to the design and implementation of the study and manuscript preparation

\section{Acknowledgments}

We thank the Molecular Biology and the Flow Cytometry and Imaging core facilities at the Stowers Institute for assistance. We are grateful to members of Rong Li lab, particularly Jin Zhu, for insightful discussions. We thank Laura Blunk for her essential assistance with mouse chromosome sorting. This work was supported by the NIH grants RO1-GM059964 and R21-NS087485 to Rong Li. Tamara Potapova was supported by a Postdoctoral Fellowship, PF-12-129-01-CCG from the American Cancer Society. This paper is subject to the NIH Public Access Policy.

\section{Competing interests}

Patent application(s) may be pending.

\section{References}

1. Dayakar, S., D.S. Rani, S.J. Babu, K. Srilatha, U. Jayanthi, K.I. Goud, D. Jain, and V. Raina. 2010. Increasing role of cytogenetics in pediatric practice. Genetic testing and molecular biomarkers 14:197-204

2. Wang, J.-C.C. 2010. The Principles of Clinical Cytogenetics (Chapter 8 - Autosomal Aneuploidy) Humana Press Inc., Totowa, NJ
3. Hanahan, D. and R.A. Weinberg. 2011. Hallmarks of cancer: the next generation. Cell 144:646-674.

4. Hanahan, D. and R.A. Weinberg. 2000. The hallmarks of cancer. Cell 100:57-70.

5. Heng, H.H., G. Liu, J.B. Stevens, B.Y. Abdallah, S.D. Horne, K.J. Ye, S.W. Bremer, S.K. Chowdhury, and C.J. Ye. 2013. Karyotype heterogeneity and unclassified chromosomal abnormalities. Cytogenet Genome Res 139:144-157.

6. Heng, H.H., G. Liu, J.B. Stevens, S.W. Bremer, K.J. Ye, B.Y. Abdallah, S.D. Horne, and C.J. Ye. 2011. Decoding the genome beyond sequencing: the new phase of genomic research. Genomics 98:242-252.

7. Imataka, G. and O. Arisaka. 2012. Chromosome analysis using spectral karyotyping (SKY). Cell Biochem Biophys 62:13-17.

8. Schrock, E., T. Veldman, H. Padilla-Nash, Y. Ning, J. Spurbeck, S. Jalal, L.G. Shaffer, P. Papenhausen, et al. 1997. Spectral karyotyping refines cytogenetic diagnostics of constitutional chromosomal abnormalities. Hum Genet 101:255-262.

9. Trask, B.J. 2002. Human cytogenetics: 46 chromosomes, 46 years and counting. Nat Rev Genet 3:769778.

10. Speicher, M.R., S. Gwyn Ballard, and D.C. Ward. 1996. Karyotyping human chromosomes by combinatorial multi-fluor FISH. Nat Genet 12:368-375.

11. Jalal, S.M. and M.E. Law. 1999. Utility of multicolor fluorescent in situ hybridization in clinical cytogenetics. Genet Med 1:181-186.

12. Schrock, E., S. du Manoir, T. Veldman, B. Schoell, J. Wienberg, M.A. Ferguson-Smith, Y. Ning, D.H. Ledbetter, et al. 1996. Multicolor spectral karyotyping of human chromosomes. Science 273:494497.

13. Rabbitts, P., H. Impey, A. Heppell-Parton, C. Langford, C. Tease, N. Lowe, D. Bailey, M. Ferguson-Smith, and N. Carter. 1995. Chromosome specific paints from a high resolution flow karyotype of the mouse. Nat Genet 9:369-375.

14. Telenius, H., A.H. Pelmear, A. Tunnacliffe, N.P. Carter, A. Behmel, M.A. Ferguson-Smith, M. Nordenskjold, R. Pfragner, and B.A. Ponder. 1992. Cytogenetic analysis by chromosome painting using DOP-PCR amplified flow-sorted chromosomes. Genes Chromosomes Cancer 4:257-263.

15. Gribble, S., B.L. Ng, E. Prigmore, D.C. Burford, and N.P. Carter. 2004. Chromosome paints from single copies of chromosomes. Chromosome Res 12:143-151.

16. Fujiwara, T., M. Bandi, M. Nitta, E.V. Ivanova, R.T. Bronson, and D. Pellman. 2005. Cytokinesis failure generating tetraploids promotes tumorigenesis in p53-null cells. Nature 437:1043-1047.

17. Ganem, N.J., Z. Storchova, and D. Pellman. 2007. Tetraploidy, aneuploidy and cancer. Curr Opin Genet Dev 17:157-162.

18. Carter, S.L., K. Cibulskis, E. Helman, A. McKenna, H. Shen, T. Zack, P.W. Laird, R.C. Onofrio, et al. 2012. Absolute quantification of somatic DNA alterations in human cancer. Nat Biotechnol 30:413-421.

19. Dewhurst, S.M., N. McGranahan, R.A. Burrell, A.J. Rowan, E. Gronroos, D. Endesfelder, T. Joshi, D. Mouradov, et al. 2014. Tolerance of whole-genome doubling propagates chromosomal instability and accelerates cancer genome evolution. Cancer discovery 4:175-185.

20. de Bruin, E.C., N. McGranahan, R. Mitter, M. Salm, D.C. Wedge, L. Yates, M. Jamal-Hanjani, S. Shafi, et al. 2014. Spatial and temporal diversity in genomic instability processes defines lung cancer evolution. Science 346:251-256.
21. Zack, T.I., S.E. Schumacher, S.L. Carter, A.D. Cherniack, G. Saksena, B. Tabak, M.S. Lawrence, C.Z. Zhang, et al. 2013. Pan-cancer patterns of somatic copy number alteration. Nat Genet 45:1134-1140.

22. Bodnar, A.G., M. Ouellette, M. Frolkis, S.E. Holt, C.P. Chiu, G.B. Morin, C.B. Harley, J.W. Shay, et al. 1998. Extension of life-span by introduction of telomerase into normal human cells. Science 279:349-352.

23. Rehen, S.K., M.J. McConnell, D. Kaushal, M.A. Kingsbury, A.H. Yang, and J. Chun. 2001. Chromosomal variation in neurons of the developing and adult mammalian nervous system. Proc Natl Acad Sci U S A 98:13361-13366.

24. Rehen, S.K., Y.C. Yung, M.P. McCreight, D. Kaushal, A.H. Yang, B.S. Almeida, M.A. Kingsbury, K.M. Cabral, et al. 2005. Constitutional aneuploidy in the normal human brain. J Neurosci 25:2176-2180.

25. Yurov, Y.B., I.Y. Iourov, V.V. Monakhov, I.V. Soloviev, V.M. Vostrikov, and S.G. Vorsanova. 2005. The variation of aneuploidy frequency in the developing and adult human brain revealed by an interphase FISH study. J Histochem Cytochem 53:385-390

26. Yurov, Y.B., I.Y. lourov, S.G. Vorsanova, T. Liehr, A.D. Kolotii, S.I. Kutsev, F. Pellestor, A.K. Beresheva, et al. 2007. Aneuploidy and confined chromosomal mosaicism in the developing human brain. PLoS One 2:e558.

27. McConnell, M.J., M.R. Lindberg, K.J. Brennand, J.C. Piper, T. Voet, C. Cowing-Zitron, S. Shumilina, R.S. Lasken, et al. 2013. Mosaic copy number variation in human neurons. Science 342:632-637.

28. Barretina, J., G. Caponigro, N. Stransky, K. Venkatesan, A.A. Margolin, S. Kim, C.J. Wilson, J. Lehar, et al. 2012. The Cancer Cell Line Encyclopedia enables predictive modelling of anticancer drug sensitivity. Nature 483:603-607.

29. Pang, A.W., J.R. MacDonald, D. Pinto, J. Wei, M.A. Rafiq, D.F. Conrad, H. Park, M.E. Hurles, et al. 2010. Towards a comprehensive structural variation map of an individual human genome. Genome Biol 11:R52.

30. Speicher, M.R. and N.P. Carter. 2005. The new cytogenetics: blurring the boundaries with molecular biology. Nat Rev Genet 6:782-792.

31. Le Scouarnec, S. and S.M. Gribble. 2012. Characterising chromosome rearrangements: recent technical advances in molecular cytogenetics. Heredity (Edinb) 108:75-85.

32. Metzker, M.L. 2010. Sequencing technologies - the next generation. Nat Rev Genet 11:31-46.

33. Navin, N.E. 2014. Cancer genomics: one cell at a time. Genome Biol 15:452.

34. Knouse, K.A., J. Wu, C.A. Whittaker, and A. Amon. 2014. Single cell sequencing reveals low levels of aneuploidy across mammalian tissues. Proc Natl Acad Sci U S A 111:13409-13414.

Received 07 April 2015; accepted 05 August 2015.

Address correspondence to Tamara A. Potapova, Stowers Institute for Medical Research, 1000 E. 50 ${ }^{\text {th }}$ Street, Kansas City, MO or Rong Li, Department of Cell Biology, Johns Hopkins University School of Medicine, 855, N. Wolfe Street, Baltimore, MD 21205. E-mail: tpo@stowers.org or rong@jhu.edu

To purchase reprints of this article, contact: biotechniques@fosterprinting.com 